\title{
Developing Systems to Measure and Monitor Resistance to ALS inhibiting herbicides in the USA.
}

\author{
Dale Shaner \\ American Cyanamid Company,Princeton, \\ NJ 08543-0400,USA
}

Resistance to acetolactate synthase (ALS) inhibiting herbicides appeared in the U.S. in 1987 with the selection of chlor sulfuron resistance in prickly lettuce. At present there are approximately 56 biotypes with resistance to various ALS inhibitors found throughout the world. With the appearance of resistance to ALS inhibitors, a means for measuring and verifying resistance was needed. Multiple methods are available for determining if a biotype have been developed. These include traditional whole plant screening, in vitro assays, and labor atory analysis.

\section{Whole Plant Screening}

The most definitive and least ambiguous method for determining if a weed biotype is resistant to an ALS inhibitor is through whole plant assays. In such assays, plants from the test population are grown from seed and compared to a known susceptible population. It is important in this type of testing to collect seed in the appropriate manner from the test population. One should 1)collect seeds when the majority are mature; 2)Collect over an area of at least $100 \mathrm{~m}$ by $50 \mathrm{~m} ; 3$ )Collect high quality seed; 4)Air dry the seed as soon as possible; and 5) Clean samples to remove poor quality seed. It is important during the test to always include a reference susceptible population and to include enough replications to allow statistical analysis. It is also best to use a range of rates to obtain a dose response curve rather than rely on a single dose assay. If a single dose is used, be sure to use a dose high enough to adequately discriminate between true resistance and potential escapes. This dose can only be arrived at through extensive testing of known populations.

\section{In vitro ass ay}

Enzyme assays Most, but not all, resistance to ALS inhibitors is due to an alteration at the site of action, ALS. Thus, one can of ten use an in vitro enzyme assay to determine if a plant population is resistant to an ALS inhibitor or not. This type of assay has advantages over whole plant screening in terms of time and space. However, such assays have to be done carefully in or der to avoid artifacts. The source of the plant material from which the enzyme is extracted is critical to the success of in vitro enzyme assays. Young, rapidly growing tissue is the best source. It is difficult, if not impossible, to use plant mater ial that has been shipped from the field to a laboratory. It is best if the plant material is from young 
seedlings grown from seeds of the test population. Extracting fully elongated, mature leaves will result in little, if any, ALS activity. In addition, senescing tissue contains an ALS-like activity that appears to be resistant to ALS inhibitors, but that has nothing to do with the site of action of these herbicides. It is critical for the tester to have experience in extracting and running an in vitro ALS assay in or der to get reliable results. In running the assay following standard protocols, one should include an extract from a susceptible control. In addition, one should include a positive control, such as valine plus leucine in order to eliminate the presence of non-ALS activity, thus avoiding artifacts.

Modified ALS assay: Another method exists that allows one to measure ALS activity in a semi-in vivo manner. In this assay, one treats the test plants with an inhibitor of ketoacid reducto isomerase (KARI), which causes an accumulation of acetolactate in plant tissue. If one compares a plant is treated with a KARI inhibitor alone with a plant treated with the KARI inhibitor plus an ALS inhibitor one can determine if the ALS inhibitor is working or not. Acetolactate can be easily ex tracted from plant material in water. Tissue treated with a KARI inhibitor alone for 6-24 hours will have large amount of acetolactate while a susceptible plant treated with a KARI inhibitor plus an ALS inhibitor will not. A resistant plant will accumulate the same or similar levels of acetolactate in the presence or absence of the ALS inhibitor. This assay can be used in the field with the appropriate equipment and can provide a relatively rapid way to determine if a plant population is resistant or not. However, the choice of plant material that is tested is critical for success and appropriate controls must be used.

Callus formation: Other methods have been developed to determine if a plant population is resistant to an ALS inhibitor. One such method is to take explants from the test population and put them on callus producing medium containing an ALS inhibitor. Plant material from a susceptible population will not produce callus in the presence of an ALS inhibitor whereas a resistant explant will produce callus. This assay can allow one to take plant material from a number of plants from a test population and determine the if the plants are resistant or not. It can be very cost effective and relatively rapid, although it has not been used to a large extent.

Molecular Biological Assays: Another method to determine if a population is resistant to an ALS inhibitor is to use the polymnerase chain reaction to pull out the ALS gene and determine if a mutation has occur red in one of the "hot spots" within the genome. Restriction analysis has also been successful. These types of assays have been successfully done under labor atory conditions, but have not been widely used to assay field populations.

Examples of these different assays will be presented and a comparison of their strengths and weaknesses will be discussed. 